\title{
Collective damage growth controls fault orientation in quasi-brittle compressive failure
}

\author{
Véronique Dansereau, ${ }^{1}$ Vincent Démery, ${ }^{2,3}$ Estelle Berthier, ${ }^{4,5}$ Jérôme Weiss ${ }^{6}$ and Laurent Ponson ${ }^{4}$ \\ ${ }^{1}$ Nansen Environmental and Remote Sensing Center, N-5006 Bergen, Norway \\ ${ }^{2}$ Gulliver, CNRS, ESPCI Paris, PSL Research University, 10 rue Vauquelin, 75005 Paris, France \\ ${ }^{3}$ Univ Lyon, ENS de Lyon, Univ Claude Bernard Lyon 1, \\ CNRS, Laboratoire de Physique, F-69342 Lyon, France \\ ${ }^{4}$ Institut Jean Le Rond d'Alembert (UMR 7190), \\ CNRS, Sorbonne Universités, 75005 Paris, France \\ ${ }^{5}$ Department of Physics, North Carolina State University, Raleigh, North Carolina 27607, USA \\ ${ }^{6}$ Univ. Grenoble Alpes, CNRS, ISTerre, 38000 Grenoble, France
}

\begin{abstract}
The Mohr-Coulomb criterion is widely used in geosciences and solid mechanics to relate the state of stress at failure to the observed orientation of the resulting faults. This relation is based on the assumption that macroscopic failure takes place along the plane that maximizes the Coulomb stress. Here, this hypothesis is assessed by simulating compressive tests on an elasto-damageable material that follows the Mohr-Coulomb criterion at the mesoscopic scale. We find that the macroscopic fault orientation is not given by the Mohr-Coulomb criterion. Instead, for a weakly disordered material, it corresponds to the most unstable mode of damage growth, which we determine through a linear stability analysis of its homogeneously damaged state. Our study reveals that compressive failure emerges from the coalescence of damaged clusters within the material and that this collective process is suitably described at the continuum scale by introducing an elastic kernel that describes the interactions between these clusters.
\end{abstract}

In 1773, Charles-Augustin de Coulomb proposed his celebrated failure criterion for materials loaded under shear or compression [1]. He postulated that failure occurs along a fault plane when the applied shear stress $\tau$ acting on that plane overcomes a resistance consisting of two parts of different nature: a cohesion $\tau_{c}$, which can be interpreted as an intrinsic shear strength of the material, and a resistance proportional to the normal pressure, $\sigma_{N}$. This results in the Mohr-Coulomb (MC) failure criterion:

$$
|\tau|=\tau_{c}+\mu \sigma_{N} .
$$

Following the former work of Amontons [2], this dependence upon pressure led Coulomb to call it friction, with $\mu$, the corresponding friction coefficient and $\phi=\tan ^{-1}(\mu)$, the angle of internal friction. As a consequence, faulting should occur along the plane that maximizes the Coulomb's stress $|\tau|-\mu \sigma_{N}$. Its orientation with respect to the maximum principal compressive stress is given by the $\mathrm{MC}$ angle

$$
\theta_{\mathrm{MC}}=\frac{\pi}{4}-\frac{\phi}{2} .
$$

This work led to the so-called Anderson theory of faulting [3], which is widely used in geophysics to interpret the orientation of conjugate faults [4] and the orientation of faults with respect to tectonic forces [5]. In this theory, $\theta_{\mathrm{MC}}$ is uniquely a function of the internal friction angle $\phi$ and hence is independent of confinement and dilatancy.

Solid mechanics models of compressive failure generally adopt the same point of view: fault formation is described as a localization instability in the constitutive inelastic response of the material $[6,7]$. As such, if the material behavior follows the Mohr-Coulomb criterion, the fault inclination observed at the macroscopic scale is expected to follow the MC angle prediction (2).

However, important issues remain to be addressed regarding the applicability of this theory. Even though the MC criterion (1) describes accurately the failure enveloppe of quasi-brittle solids like rocks $[8,9]$ and ice $[10,11]$, the ability of MC angle prediction (2) to capture fault orientation is still debated $[12,13]$. In particular, experiments have reported an increase of the fault angle with the lateral confinement, which is incompatible with the MC prediction [14-16]. Besides, while Coulomb's theory provides a simple instantaneous criterion for failure, it says nothing about the process of damage spreading that precedes it. It is now widely accepted that the compressive failure of quasi-brittle materials does not occur suddenly, but instead involves the nucleation and growth of microcracks, which interact and finally coalesce to form a macroscopic fault [17-19]. It is not clear at all if this phenomenology is compatible with the point of view that macroscopic faulting emerges from a local instability in the material constitutive response $[3,6,7]$, nor with the assumption that fault orientation in materials that do follow the $\mathrm{MC}$ failure criterion is given by the $\mathrm{MC}$ angle.

Damage spreading under compression and the progression towards macroscopic failure is well captured by continuum damage models, wherein microcrack density at the mesoscopic scale is represented by a damage variable and is coupled to the elastic modulus of the material [20-23] (Fig. 1). In these models, a failure criterion is implemented at the local scale, that is, usually, the scale of the mesh grid element. Material heterogeneity is accounted for by introducing some noise in either the elastic modulus or the local failure criterion. When the state of 
stress over a given element exceeds this criterion, the level of damage of this element increases, thereby decreasing its elastic modulus. Long-range elastic interactions arise from the stress redistribution initiated by the local drop in the elastic modulus. This redistribution can induce damage growth in neighboring elements and eventually trigger avalanches of damaging events over longer distances. Such models have been shown to reproduce many features of brittle compressive failure, such as the clustering of rupture events and the power law distribution of acoustic events sizes prior to the emergence of a macroscopic fault [20, 24-26]. They are thus relevant tools to study the process of damage localization that leads to failure and, in particular, the dependence of the angle of localization of damage on the parameters involved in the damage criteria.

Here, we use such a tool to investigate how the macroscopic fault emerges from the accumulation of microscopic damage events and test commonly used models that describe compressive failure as a local material instability [6, 7]. In particular, we simulate compression experiments of specimens of an elasto-damageable material that satisfy the MC failure criterion at the mesoscopic scale and study the inclination of the macroscopic rupture plane as a function of the internal friction angle under different confinement conditions. We show that the orientation of the simulated fault is not given by the MC angle. Instead, we find that the most unstable mode of damage growth, which is inferred from a linear stability analysis at the specimen scale, provides a good estimation of the fault orientation for weakly heterogeneous materials. Our findings shed light on the significance of elastic interactions and damage coalescence on the fault formation during compressive failure of quasi-brittle materials. It also suggests that the modeling strategy that consists in damage localization from the homogenized material response may be insufficient, but that this difficulty may be overcome by addressing the stability of the damage growth process at the macroscopic scale using the elastic interaction kernel introduced in this study.

Following Refs. [22, 24] and others, the model is based on an isotropic linear-elastic constitutive law where the elastic modulus,

$$
E(d)=(1-d) E^{0}
$$

is a decreasing function of the scalar internal variable, $d \in[0,1]$, which describes the level of damage in a material element, with $E^{0}$ the Young's modulus of the undamaged specimen. For sake of simplicity, Poisson's ratio, $\nu$, is assumed constant and does not vary with $d$. Material heterogeneities are introduced via the local critical strength by assigning different cohesions, $\tau_{\mathrm{c}}$, to the constitutive material elements.

In the numerical simulations, a two-dimensional rectangular specimen of an elasto-damageable material with dimensions $L \times L / 2$ is compressed with a stress $\Sigma_{1}$ by
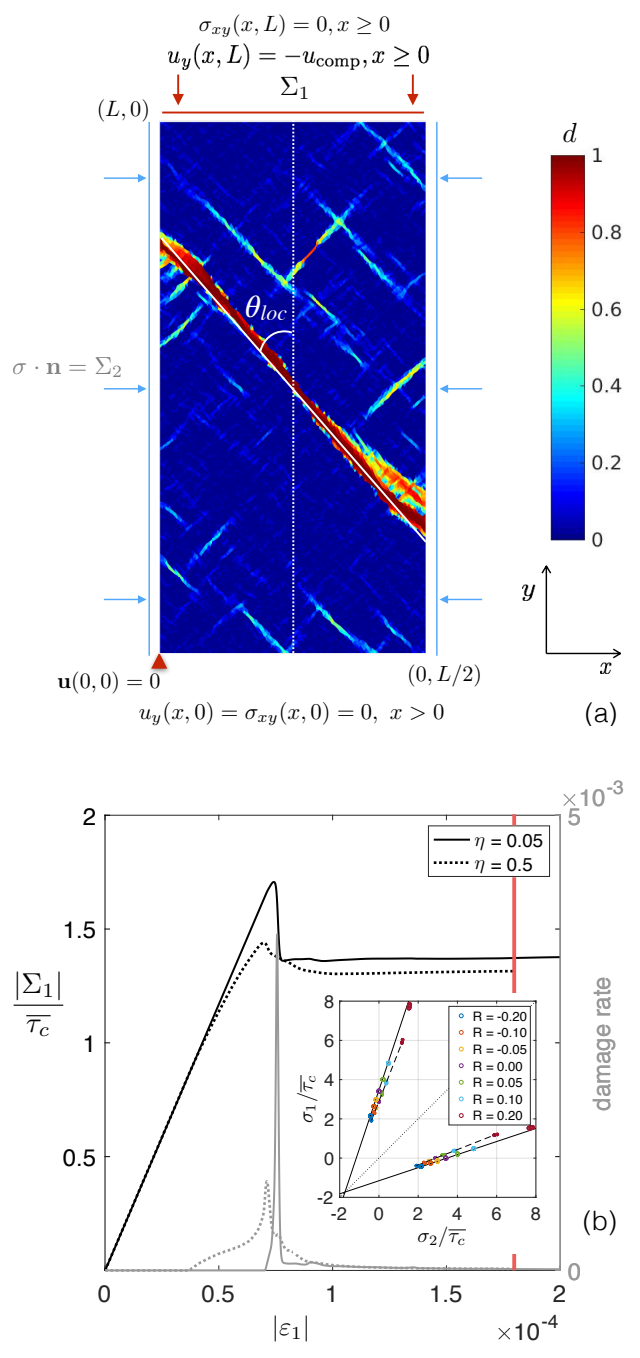

FIG. 1. Compressive test simulation: (a) The prescribed boundary conditions are superimposed to a snapshot of the field of the level of damage, $d$, simulated after peak load (timing indicated by the red vertical line in (b)). The material properties in this simulation are $\phi=30^{\circ}$ and $\nu=0.3$ and the disorder parameters, $\eta=0.05$ and $a=1$. No lateral confinement is applied. The orientation of the fault, $\theta_{\text {loc }}$, is determined by a projection histogram method [16]. (b) The corresponding stress-strain (black) and damage rate (grey) curves are given by the solid lines. The dotted lines show the same quantities for a simulation using identical loading and material properties and a stronger disorder $(\eta=0.5$ and $a=1$ ). (b, inset) Macroscopic maximum and minimum principal stresses, $\Sigma_{1}, \Sigma_{2}$, (colored dots) estimated at the onset of damage localization (i.e. at peak load) in a set of 5 simulations using the same material properties as in (a) and (b) and different confining ratios (biaxial compression for $R>0$ and biaxial compression-tension for $R<0$ ). The black solid lines represent the $\mathrm{MC}$ criterion for $\overline{\tau_{c}}=1$. Open circles are used for the disorder parameters $\eta=0.05$ and $a=1$ and filled circles for the parameters $\eta=0.5$ and $a=1$. 
prescribing a constant velocity, $u_{\text {comp }}$, on its upper short edge with the opposite edge fixed in the direction of the forcing (Fig. 1a). Plane stresses are assumed. A confining stress $\Sigma_{2}$ can be applied on the lateral sides; in this case, the confinement ratio $R=\Sigma_{2} / \Sigma_{1}$ is kept constant. We denote $\boldsymbol{\sigma}^{0}$ the external stress tensor prescribed to the sample. At each time step, the damage level of the material elements for which the stress is over-critical with respect to the local $\mathrm{MC}$ criterion is increased such that overcritical stresses are projected back onto the $\mathrm{MC}$ enveloppe [16]. Both the prescribed velocity on the upper edge of the specimen and the lateral confinement are small enough to ensure a quasi-static driving and small deformations. The simulations therefore rely on the numerical resolution of the following force balance and Hooke's law:

$$
\begin{aligned}
\nabla \cdot \boldsymbol{\sigma}(\boldsymbol{r}) & =0 \\
\boldsymbol{\sigma}(\boldsymbol{r}) & =\frac{E}{1+\nu} \boldsymbol{\epsilon}(\boldsymbol{r})+\frac{E \nu}{1-\nu^{2}} \operatorname{tr}(\boldsymbol{\epsilon}(\boldsymbol{r})) \mathbf{1},
\end{aligned}
$$

where $\boldsymbol{\sigma}(\boldsymbol{r})$ and $\boldsymbol{\epsilon}(\boldsymbol{r})$ are the planar stress and strain tensors in the specimen.

Equations (4) and (5) are solved using variational methods on a 2-dimensional amorphous grid made of more than 33000 triangular elements [16]. A typical stress-strain response is shown in Fig. 1(b) for no confinement, $\phi=30^{\circ}$ and $\nu=0.3$. Consistent with the failure in compression of quasi-brittle materials monitored via acoustic emissions $[26,27]$ as well as with previous progressive damage simulations of this process [22], the simulated damage indicates some precursory activity. It is initially distributed homogeneously over the domain (not shown) and localizes progressively as the loading is increased. Fault formation is identified by the sudden rise of the damage rate and corresponds to peak load.

As done in laboratory experiments on rocks [13, 15] and ice [10], we measured the failure enveloppe by testing specimens under different confinement ratios (see inset of Fig. 1(b)). We observe that the failure enveloppe of the specimen given by the principal stresses $\left(\Sigma_{1}, \Sigma_{2}\right)$ at peak load reproduces the MC criterion enforced at the material level. Therefore, in agreement with observations [11], $\mu$ appears to be a scale-independent property in our numerical model.

The damage field after peak load exhibits a localization band characteristic of compressive failure (Fig. 1(a)). A projection histogram method is used to determine its orientation [16], hereinafter referred to as the localization angle, $\theta_{\text {loc. }}$. We observe that the value of $\theta_{\text {loc }}$ is robust and independent of both the mesh size and the aspect ratio of the specimen [16]. A first set of compression test simulations representing a minimum disorder scenario is initialized with a field of cohesion that is uniform for all except one element chosen at random. For this inclusion, $\tau_{c}$ is initially $5 \%$ weaker and is reset to the uniform value of its neighbors after its first damage event. Fig. 2(a) shows the mean localization angle as a function of the internal friction angle $\phi$ and Fig. 2(b,c), the same results for different Poisson's and confinement ratios, respectively. Neither the value nor the variation of $\theta_{\text {loc }}$ with $\phi$ agree with the MC prediction. In particular, the simulated fault orientation varies with Poisson's ratio as well as with confinement, a dependence that is not accounted for in the MC theory, but that has been observed in laboratory experiments on rocks [13-15].

To understand how macroscopic failure arises in the model, we perform a linear stability analysis of the homogeneously damaged solution. In our simulations, the damage field follows the evolution law

$$
\frac{\partial d}{\partial t}(\boldsymbol{r}, t)=F\left[\boldsymbol{\sigma}^{0}, d(\boldsymbol{r}, t)\right]
$$

where the damage driving force $F$ is non-local: its value for a material element depends on the damage level everywhere in the specimen. The linear stability analysis amounts to linearizing this evolution equation around an homogeneous damage field. Assuming an infinite specimen, the problem is translation invariant and the linearization can be written as a convolution product of the damage field with the elastic kernel $\Psi_{\sigma^{0}, d^{0}}[28]$ :

$$
F\left[\boldsymbol{\sigma}^{0}, d(\boldsymbol{r}, t)\right] \simeq F\left[\boldsymbol{\sigma}^{0}, d^{0}\right]+\Psi_{\boldsymbol{\sigma}^{0}, d^{0}} * \delta d(\boldsymbol{r}, t),
$$

where $\delta d(\boldsymbol{r}, t)=d(\boldsymbol{r}, t)-d^{0} \ll 1$. The kernel $\Psi$ is reminiscent of the Eshelby solution for the mechanical field around a soft inclusion embedded in an infinite 2D elastic medium, which also decays as $1 / r^{2}$ [29]. It provides the redistribution of driving force $F$ following a localized ( $\delta$-distributed) damage growth and as such, describes the elastic interactions between material elements during damage spreading. In Fourier space, it does not depend on the magnitude of the wavevector $\boldsymbol{q}$, but only on its polar angle, $\omega[16]$ :

$$
\tilde{\Psi}(\omega)=A\left[\sin (\omega)^{2}-\frac{1+\sin (\phi)}{2}\right] \times\left[\delta-\sin (\omega)^{2}\right]
$$

with $A=2 \Sigma_{1} \frac{(1-\nu)(1-R)}{1-d^{0}}$ and $\delta=\frac{\nu-R}{(1+\nu)(1-R)}$. The evolution of the damage field perturbations is inferred from Eqs. $(6,7)$. Considering harmonic modes $\delta d(\boldsymbol{r}) \propto \cos (\boldsymbol{q}$. $\boldsymbol{r})$, their growth rate is given by $\tilde{\Psi}(\omega)$. Since the kernel is maximal and positive for $\sin \left(\omega^{*}\right)^{2}=[1+\sin (\phi)+2 \delta] / 4$, one concludes that (i) a homogeneous damage field is unstable and (ii) all the wavevectors with the orientation $\omega^{*}$ diverge at the same rate as $\tilde{\Psi}$ is independent of the magnitude of the wavevector. Hence, any linear combination of these modes also diverges at the same rate, corresponding to a localization band that is perpendicular to $\boldsymbol{q}$, leading to an inclination $\theta_{\mathrm{LS}}=\pi / 2 \pm \omega^{*}$ or

$$
\theta_{\mathrm{LS}}=\arccos \left(\frac{\sqrt{1+\sin (\phi)+2 \delta}}{2}\right)
$$



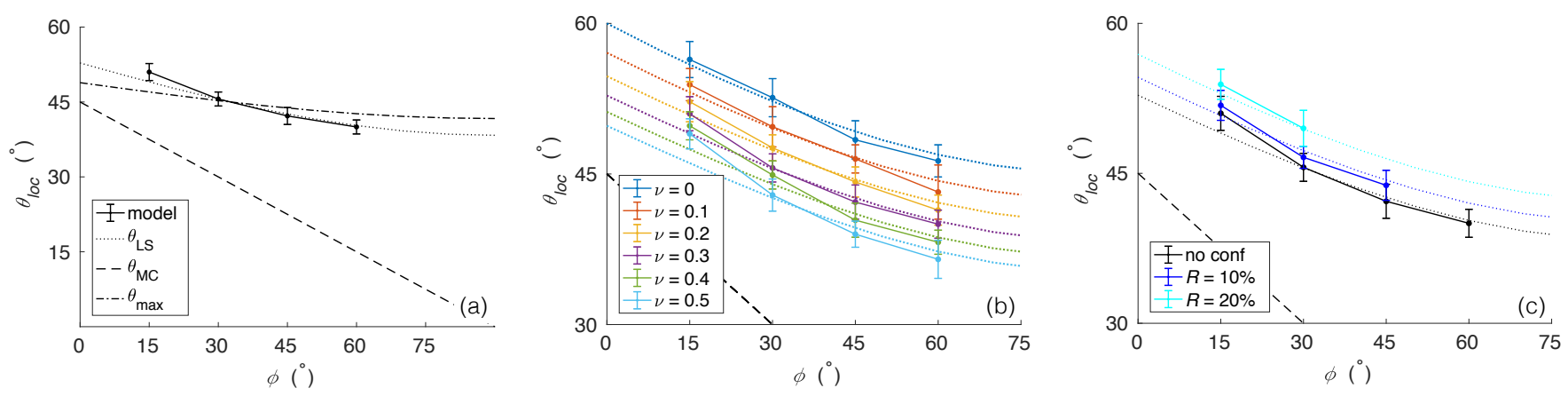

FIG. 2. (a) Mean localization angle $\theta_{\text {loc }}$ as a function of the internal friction angle $\phi$ for an ensemble of 25 simulations with minimal disorder using identical boundary and loading conditions. No confinement is applied and $\nu=0.3$. The black dashed line shows the $\mathrm{MC}$ prediction, $\theta_{\mathrm{MC}}$, the dotted line, the angle of the most unstable mode, $\theta_{\mathrm{LS}}$ and the dashed-dotted line, the angle of maximal stress redistribution, $\theta_{\max }$. The error bars represent \pm 1 standard deviation from the mean. Mean localization angle for (b) different values of Poisson's ratio without confinement and (c) different values of confinement ratio for $\nu=0.3$.

with respect to the direction of maximum principal compressive stress. For the sake of simplicity, only the solution lying in $[0, \pi / 2]$ is kept here, but both inclinations are actually possible in agreement with the orientation of the secondary faults observed in Fig. 1(a).

We compare the predicted inclination, $\theta_{\mathrm{LS}}$, with the localization angle, $\theta_{\mathrm{loc}}$, from the simulations. We find that the prediction is in excellent agreement with the results of the minimal disorder numerical simulations (Fig. 2(a)) and reproduces the observed dependence on Poisson's ratio (Fig. 2(b)). The increase of $\theta_{\text {loc }}$ with confinement (Fig. 2(c)) is also well captured, in qualitative agreement with experimental observations [15, 16].

Alternatively, the fault orientation may be compared to the direction along which stress redistribution is maximal after a damage event [30]. This angle, $\theta_{\max }=$ $\arccos (\sqrt{[3+\sin (\phi)+2 \delta] / 8})$, which maximizes the angular part of the elastic kernel in real space [16], is significantly different from the orientation of the most unstable mode, $\theta_{\text {LS }}$. Recent compression experiments on granular materials $[31,32]$ have suggested that $\theta_{\max }$ may correspond to the preferred orientation of the precursory damage cascades prior to failure while $\theta_{\mathrm{LS}}$ provides the final macroscopic fault inclination. As shown in Fig. 2(a), $\theta_{\mathrm{LS}}$ clearly provides a better agreement with the simulations than $\theta_{\max }$ in the case of a single evanescent heterogeneity.

Real and, especially, natural materials are heterogeneous and comprise many randomly distributed impurities that can serve as local stress concentrators, initiating microcracking and leading to an extended regime of diffuse damage growth prior to localization [17, 19, 33, 34]. To determine if and how this regime affects the final orientation of the macroscopic fault, we introduce disorder in the critical strength by drawing randomly the cohesion, $\tau_{c}$, of a proportion $a$ of the material elements in the range $\bar{\tau}_{\mathrm{c}}[1-\eta, 1+\eta]$, with the cohesion of the remaining proportion $1-a$ of the elements set to the average cohesion, $\bar{\tau}_{\mathrm{c}}$. We consider cases of weak $(\eta=0.05$, Fig. $3(\mathrm{a}))$ and strong $(\eta=0.5$, Fig. 3(b)) disorder. In both cases, the value of $a$ is varied between $10^{-4}$, corresponding to a few $(\simeq 3)$ inclusions in a homogeneous matrix, and $a=1$, for which all elements have a different critical strength. Consistent with the minimum disorder case investigated above, the agreement with the orientation obtained from the linear stability analysis, $\theta_{\mathrm{LS}}$, is best for $a=10^{-4}$ (Fig. $3(\mathrm{a}, \mathrm{b})$ ). The deviation from $\theta_{\mathrm{LS}}$ increases with both the density $a$ of inclusions and the strength $\eta$ of the disorder, indicating that disorder significantly affects the fault orientation $\theta_{\mathrm{loc}}$. In all cases however, $\theta_{\text {loc }}$ remains well above $\theta_{\mathrm{MC}}$, and a clear dependence on Poisson's ratio and on confinement is still observed (see Fig. 3(c, d)). These departures from the MC theory are in qualitative agreement with the experimental observations reporting the localization angle and its dependence on confinement [10,13-16]. As a direct consequence, our findings question the estimation of internal friction or of applied stresses from faults orientation in natural settings [3-5]. To go further in the comparison of experimental observations with the newly developed theory, triaxial loading as well as a possible dependence of Poisson's ratio on damage should be introduced.

To conclude, the discrepancy between the fault angle and the Mohr-Coulomb prediction indicates that compressive failure, even when it is not preceded by an extended regime of stable damage growth, results from the collective spreading of damage within the specimen. As such, the fault angle observed in our simulations is successfully captured from a stability analysis performed at the macroscopic scale. The role of elasticity, which is responsible for the redistribution of the stress after a damage event and for interactions between microcracks, reflects in the dependence of the localization angle on the Poisson's ratio. The fact that the MC criterion, derived from the stability of a single material element, fails to 

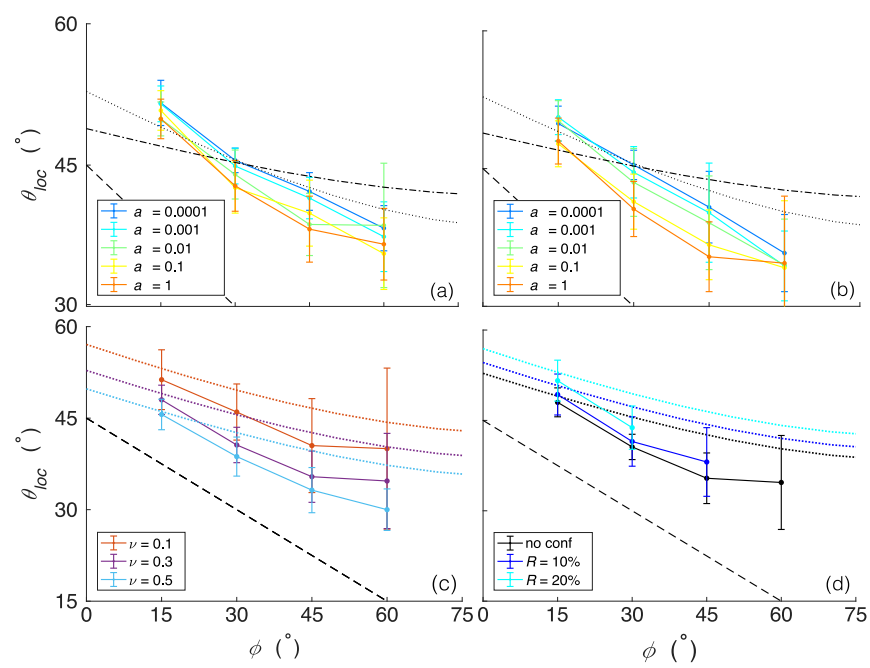

(b)

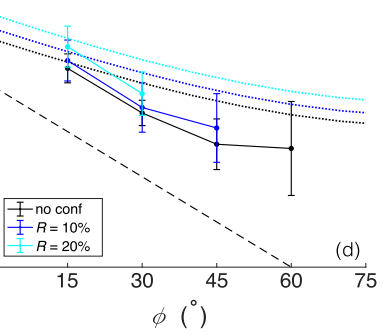

FIG. 3. Localization angle measured from the compression simulations as a function of the internal friction angle $\phi$ for (a) weak disorder $(\eta=0.05)$ and (b) strong disorder $(\eta=0.5)$ and different values of $a$. No confinement is applied and $\nu=0.3$. Mean $\theta_{\mathrm{loc}}$ for $a=1$ and $\eta=0.5$ (strong disorder) and (c) different values of $\nu$ without confinement and (d) different confinement ratios for $\nu=0.3$. The maximum confinement ratio, $R_{\max }$ [16], is $58 \%$ for $\phi=15^{\circ}, 33 \%$ for $\phi=30^{\circ}, 17 \%$ for $\phi=45^{\circ}$ and $7 \%$ for $\phi=60^{\circ}$. The black dashed line shows $\theta_{\mathrm{MC}}$, the dotted lines, $\theta_{\mathrm{LS}}$, and the dashed-dotted line, $\theta_{\max }$.

predict the fault angle suggests commonly used modeling approaches to compressive failure $[6,7]$ that do not account for the long-range elastic interactions between damage events may not predict accurately the localization threshold, the resulting band inclination and their relation with the material and loading parameters.

V. Dansereau has been supported by TOTAL S.A. E. Berthier and L. Ponson have been supported by the Emergence from Sorbonne Universités. We thank D. Kondo and A. Rosso for enlightening discussions and A. Amon for providing a code for the projection histogram calculations.

[1] C. A. Coulomb, "Essai sur une application des règles de maximis et minimis à quelques problèmes de statique relatifs à l'architecture," Mémoires de Mathématiques et de Physique par divers savants 7, 343-382 (1773).

[2] G. Amontons, "Mémoires de l'Académie Royale," (J. Boudot, Paris, 1699) Chap. De la résistance causée dans les machines (About resistance and force in machines), pp. 257-282.

[3] E. M. Anderson, "The dynamics of faulting," Transactions of the Edinburgh Geological Society 8, 387-402 (1905).

[4] E. M. Schulson, "Compressive shear faults within arctic sea ice: Fractures on scales large and small," J. Geophys. Res. 109 (2004), 10.1029/2003JC002108.
[5] Z. Reches, "Determination of the tectonic stress tensor from slip along faults that obey the coulomb yield condition," Tectonics 6, 849-861 (1987).

[6] J. W. Rudnicki and J. R. Rice, "Conditions for the localization of deformation in pressure-sensitive dilatant materials," J. Mech. Phys. Solids 23, 371-394 (1975).

[7] D. Bigoni, Nonlinear solid mechanics: Bifurcation theory and material instability (Cambridge University Press, 2012).

[8] James D. Byerlee, "Frictional characteristics of granite under high confining pressure," J. Geophys. Res. 72, 3639-3648 (1967).

[9] J. C. Jaeger and N. G. W. Cook, Fundamentals of Rock Mechanics (Chapman and Hall, Cambridge UK, 1979).

[10] E. M. Schulson, A. L. Fortt, D. Iliescu, and C. E. Renshaw, "On the role of frictional sliding in the compressive fracture of ice and granite: Terminal vs. post-terminal failure," Acta Mater. 54, 3923 - 3932 (2006b).

[11] J. Weiss and E. M Schulson, "Coulombic faulting from the grain scale to the geophysical scale: lessons from ice," J. Phys. D. Appl. Phys. 42, 214017 (2009).

[12] J. P. Bardet, "A comprehensive review of strain localization in elastoplastic soils," Comput. Geotech. 10, 163-188 (1990).

[13] A. Hackston and E. Rutter, "The Mohr-Coulomb criterion for intact rock strength and friction - a re-evaluation and consideration of failure under polyaxial stresses," Solid Earth 7, 493-508 (2016).

[14] A. Haied, D. Kondo, and J. P. Henry, "Strain localization in Fontainebleau sandstone," Mech. Cohes.-Frict. Mater. 5, 239-253 (2000).

[15] B. Haimson and J. W. Rudnicki, "The effect of the intermediate principal stress on fault formation and fault angle in siltstone," J. Struct. Geol. 32, 1701 - 1711 (2010), fault Zones.

[16] "See supplemental material at [url will be inserted by publisher] for more details." .

[17] D. A. Lockner, J. D. Byerlee, V. Kuksenko, A. Ponomarev, and A. Sidorin, "Quasi-static fault growth and shear fracture energy in granite," Nature 350, 39-42 (1991).

[18] J. Fortin, S. Stanchits, G. Dresen, and Y. Gueguen, eds., Rock Physics and Natural Hazards (Birkhäuser Basel, 2009) p. 823.

[19] F. Renard, B. Cordonnier, M. Kobchenko, N. Kandula, J. Weiss, and W. Zhu, "Microscale characterization of rupture nucleation unravels precursors to faulting in rocks," Earth Planet. Sc. Lett. 476, 69-78 (2017).

[20] S. Zapperi, A. Vespignani, and H. E. Stanley, "Plasticity and avalanche behaviour in microfracturing phenomena," Nature 388, 658-660 (1997).

[21] V. Lyakhovsky, Y. Ben-Zion, and A. Agnon, "Distributed damage, faulting and friction," J. Geophys. Res. 102, 27635-27649 (1997).

[22] D. Amitrano, J.-R. Grasso, and D. Hantz, "From diffuse to localised damage through elastic interaction," Geophys. Res. Lett. 26, 2109-2112 (1999).

[23] E. Berthier, V. Démery, and L. Ponson, "Damage spreading in quasi-brittle disordered solids: I. Localization and failure ," J. Mech. Phys. Solids 102, 101-124 (2017).

[24] C. Tang, "Numerical simulation of progressive rock failure and associated seismicity," Int. J. Rock Mech. Min. 34, 249-261 (1997).

[25] L. Girard, D. Amitrano, and J. Weiss, "Failure as a 
critical phenomenon in a progressive damage model," J. Stat. Mech. Theory Exp. 2010, -01013 (2010).

[26] D. Amitrano, "Brittle-ductile transition and associated seismicity: Experimental and numerical studies and relationship with the $b$ value," J. Geophys. Res. 108 (2003), 10.1029/2001JB000680.

[27] S. J. D. Cox and P. G. Meredith, "Microcrack formation and material softening in rock measured by monitoring acoustic emissions ," International Journal of Rock Mechanics and Mining Sciences \& Geomechanics Abstracts 30, 11-24 (1993).

[28] J. Weiss, L. Girard, F. Gimbert, D. Amitrano, and D. Vandembroucq, "(Finite) statistical size effects on compressive strength," P. Natl. Acad. Sci. Usa. 111, 6231-6236 (2014).

[29] J. D. Eshelby, "The Determination of the Elastic Field of an Ellipsoidal Inclusion, and Related Problems," Proc. Royal Soc. Lond. A Mat. Phys. 241, 376-396 (1957).

[30] A. Nicolas, E. E. Ferrero, K. Martens, and J. L. Barrat,
"Deformation and flow of amorphous solids: a review of mesoscale elastoplastic models," ArXiv e-prints (2017), arXiv:1708.09194 [cond-mat.dis-nn].

[31] A. Le Bouil, A. Amon, S. McNamara, and J. Crassous, "Emergence of Cooperativity in Plasticity of Soft Glassy Materials," Phys. Rev. Lett. 112, 246001 (2014).

[32] K. Karimi and J.-L. Barrat, "Correlation and shear bands in a plastically deformed granular medium," Sci. Rep. 8, 4021 (2018).

[33] Y. Tal, B. Evans, and U. Mok, "Direct observations of damage during unconfined brittle failure of carrara marble," J. Geophys. Res. Solid Earth 121, 1584-1609 (2016).

[34] F. Renard, J. Weiss, J. Mathiesen, Y. Ben-Zion, N. Kandula, and B. Cordonnier, "Critical evolution of damage toward system-size failure in crystalline rock," J. Geophys. Res. 123, 1969-1986 (2018). 